Pacific Journal of Mathematics

ON GROUPS OF EXPONENT FOUR WITH GENERATORS OF 


\title{
ON GROUPS OF EXPONENT FOUR WITH GENERATORS OF ORDER TWO
}

\author{
C. R. B. WRIGHT
}

1. If $x, y, \cdots$ are elements of a group $G$, we define the commutator $(x, y)$ of $x$ and $y$ by $(x, y)=x^{-1} y^{-1} x y$. More generally, we define $e x$ tended commutators inductively by $(x, \cdots, y, z)=((x, \cdots, y), z)$. In this paper we shall also be concerned with higher commutators of type $\left(\left(a_{1}, \cdots, a_{s}\right),\left(b_{1}, \cdots, b_{t}\right), \cdots,\left(c_{1}, \cdots, c_{r}\right)\right)$ which we denote by $\left(a_{1}, \cdots, a_{s}\right.$; $\left.b_{1}, \cdots, b_{t} ; \cdots ; c_{1}, \cdots, c_{r}\right)$. If we let $G_{i}$ be the subgroup of $G$ which is generated by all extended commutators of length $i$, (i.e., with $i$ entries), then $G_{i}$ is a characteristic subgroup of $G$, and the series $G=G_{1} \supset G_{2} \supset \ldots$ is called the lower central series of $G .{ }^{1}$

Let $G(n)(n=1,2, \cdots)$ be the freest group of exponent 4 on $n$ generators of order 2. That is, $G(n)$ is a group in which the fourth power of every element is the identity, $1, G(n)$ is generated by $n$ elements of order 2, and if $H$ is any other group with these properties, then $H$ is a homomorphic image of $G(n)$.

We prove $G(n)_{n+2}=1$. For this purpose it may be assumed, since $G(n)$ is finite ${ }^{2}$ and hence nilpotent, that $G(n)_{n+3}=1$. Moreover, it will be enough to show $\left(x_{1}, \cdots, x_{n+2}\right)=1$ for all choices of $x_{1}, \cdots, x_{n+2}$ from among the generators of $G(n)$.

2. Lemma 2.1. If $x, y, \cdots, z$ are elements of order 2 in a group of exponent 4 , then $(x, y)^{2}=1,(x, y, \cdots, z)^{2}=1$, and $(x, y, x)=1$.

Proof. Since $(x, y)=x y x y=(x y)^{2},(x, y)^{2}=1$. By induction, $(x, y, \cdots$, $z)^{2}=1$, while $(y, x)=y x y x=x(x, y) x=(x, y)(x, y, x)$, so that $(x, y, x)=$ $(y, x)^{2}=1$.

The relation $(x, y, \cdots, z)^{2}=1$ will be the justification for future substitutions and will be used without specific mention.

TheOREM 2.1. $G(2)_{3}=1$.

Proof. By Lemma 2.1, if the generators of $G(2)$ are $a$ and $b$, then $(a, b, a)=(b, a, a)=(a, b, b)=(b, a, b)=1$.

3. Lemma 3.1. If $a, b$ and $c$ are elements of order 2 in a group $G$ of exponent 4 , then

Received October 26, 1959. Presented to the Society on September 3, 1959. This work was supported by a National Science Foundation predoctoral fellowship.

1 For properties of commutators and the lower central series see Hall, [1] , Ch. 10.

2 See Sanov, [2], or Hall, [1], pp. 324-325. 


$$
\begin{aligned}
(a, b, c) & \equiv(b, c, a)(c, a, b) \bmod G_{5} \\
(a, b ; c, a)=(a, c ; b, a) & \equiv(a, c, b, a) \bmod G_{5} \\
(a, b, c, a) & \equiv(b, c, a, b)(c, a, b, c) \bmod G_{5}
\end{aligned}
$$

Proof. We may assume that $a, b$ and $c$ generate $G$. Now

$$
a b c a b c=a b a(a, c) b(b, c)=(a, b)(a, c)(a, c, b)(b, c) .
$$

Thus, modulo $G_{5},(a b c)^{2}=(a, b)(a, c)(b, c)(a, c, b)$. Hence

$1 \equiv[(a, b)(a, c)(b, c)]^{2} \bmod G_{5}$, so that, modulo $G_{5}$,

$1=(a, b)(a, c)(b, c)(a, b)(a, c)(b, c)=(a, b)(a, c)(a, b)(a, b ; b, c)(a, c)(a, c ; b, c)$,

$$
1 \equiv(a, b ; a, c)(a, b ; b, c)(a, c ; b, c) \bmod G_{5}
$$

But also

$$
\begin{aligned}
a b c & =c a(a, c) b(b, c) \\
& =b c(c, b) a(a, b)(a, c)(a, c, b)(b, c) \\
& =a b(b, a) c(c, a)(c, b)(c, b, a)(a, b)(a, c)(a, c, b)(b, c)
\end{aligned}
$$

so that $1=(b, a)(b, a, c)(c, a)(c, b)(c, b, a)(a, b)(a, c)(a, c, b)(b, c)$, and hence, $\operatorname{modulo} G_{5}$,

$$
\begin{aligned}
1 & =(b, a)(c, a)(c, b)(a, b)(a, c)(b, c)(b, a, c)(c, b, a)(a, c, b) \\
& =[(a, b)(a, c)(b, c)]^{2}(a, b, c)(b, c, a)(c, a, b) .
\end{aligned}
$$

Thus (1) is proved. Replacing $b$ by $(a, b)$ in (1) gives $(a, b, c, a)(c, a ; a, b) \equiv$ $1 \bmod G_{5}$ or (2). And (2) and (4) together give (3).

LEMMA 3.2. If $x_{1}, \cdots, x_{k}$ and a are elements of order 2 in a group $G$ of exponent 4 , then $\left(x_{1}, \cdots, x_{k}, a\right) \equiv X \bmod G_{k+2}$, where $X$ is a product of commutators of form $\left(a, y_{1}, \cdots, y_{k}\right)$ with $y_{1}, \cdots, y_{k}$ from among $x_{1}, \cdots, x_{k}$.

COROLlary. If $x_{1}, \cdots, x_{k}, z_{1}, \cdots, z_{s}$ and a are elements of order 2 in a group $G$ of exponent 4 , then

$$
\left(x_{1}, \cdots, x_{k}, a, z_{1}, \cdots, z_{s}\right) \equiv X \bmod G_{k+s+2}
$$

where $X$ is a product of commutators of form $\left(a, y_{1}, \cdots, y_{k}, z_{1}, \cdots, z_{s}\right)$ with $y_{1}, \cdots, y_{k}$ from among $x_{1}, \cdots, x_{k}$.

Proof of Lemma 3.2. Certainly the lemma and corollary are true if $k=1$. Assume for induction that both are true for $k=n-1 \geq 1$. 
Now by (1), modulo $G_{n+2},\left(x_{1}, \cdots, x_{n-1}, x_{n}, a\right)=\left(x_{1}, \cdots, x_{n-1}, a, x_{n}\right)\left(x_{1}, \cdots\right.$, $\left.x_{n-1} ; a, x_{n}\right)$. But by the inductive assumption $\left(x_{1}, \cdots, x_{n-1}, a, x_{n}\right)$ is a product of terms $\left(a, y_{1}, \cdots, y_{n-1}, x_{n}\right)$, and $\left(x_{1}, \cdots, x_{n-1} ; a, x_{n}\right)$ is a product of terms $\left(a, x_{n}, y_{1}, \cdots, y_{n-1}\right)$. The lemma and its immediate corollary follow by induction.

\section{THEOREM 3.1. $G(3)_{5}=1$.}

Proof. Let $a, b$ and $c$ be the generators of $G(3)$. Consider any commutator $C=\left(x_{1}, x_{2}, x_{3}, x_{4}, x_{5}\right)$ in arguments $a, b$ and $c$. We show $C=1$. There is no loss of generality in taking $x_{5}=a$. If $a$ does not appear again in $C$, then by Theorem $2.1, C=\left(1, x_{5}\right)=1$. If $a$ appears again, then by Lemma 3.2 and the assumption that $G(3)_{6}=1$, we may suppose $C=\left(a, x_{2}, x_{3}, x_{4}, a\right)$. By Lemma 2.1, if $a$ appears a third time, then $C=1$. Thus we may take $C=(a, b, c, b, a)$. Now $(a, b, c, b, a)=$ $(b, c, a, b, a)(c, a, b, b, a)=(b, c, a, b, a)$ by (1). Replacing $c$ by $(b, c)$ in (3) gives $(a, b ; b, c, ; a)=(b ; b, c ; a ; b)=1$, while replacing $c$ by $(b, c)$ in (2) gives $(a, b ; b, c ; a)=(b, c, a, b, a)$. Hence, $C=(a, b, c, b, a)=(b, c, a, b, a)=$ $(a, b ;, b, c ; a)=1$, and the theorem is proved.

COROLLARY 1. If $a, b$ and $c$ are elements of order 2 in a group of exponent 4, then

$$
\begin{aligned}
(a, b, c) & =(b, c, a)(c, a, b) \\
(a, b ; c, a) & =(a, b, c, a) \\
(a, b, c, a) & =(b, c, a, b)(c, a, b, c)
\end{aligned}
$$

Proof. These follow from Lemma 3.1.

CoROLlaRy 2. If $x_{1}, \cdots, x_{k}, y_{1}, \cdots, y_{s}, z_{1}, \cdots, z_{t}(s \geq 2)$ are elements of order 2 in a group $G$ of exponent 4, then

$$
\left(x_{1}, \cdots, x_{k} ; y_{1}, \cdots, y_{s} ; z_{1} ; \cdots ; z_{t}\right) \equiv A B \bmod G_{k+s+t+1}
$$

where

$$
\begin{aligned}
& A=\left(x_{1}, \cdots, x_{k} ; y_{1}, \cdots, y_{s-1} ; y_{s} ; z_{1} ; \cdots ; z_{t}\right) \\
& B=\left(x_{1}, \cdots, x_{k}, y_{s} ; y_{1}, \cdots, y_{s-1} ; z_{1} ; \cdots ; z_{t}\right)
\end{aligned}
$$

Proof. This follows from $\left(1^{\prime}\right)$.

The following corollary lists some relations for future use.

COROLlary 3. If $a, b, c, d$ and $f$ are elements of order 2 in a group $G$ of exponent 4, then 


$$
\begin{aligned}
(a, b, c, d, c) & \equiv(a, b, d, c, d) \bmod G_{6} \\
(b, c, a ; d, f, a) & \equiv 1 \bmod G_{7} \\
(a, f ; b, d, c) & \equiv(a, f, c ; b, d)(a, f ; b, d ; c) \\
(b, f, d ; a, c)(d, f, b ; a, c) & \equiv(b, d, f ; a, c) \bmod G_{8} .
\end{aligned}
$$

Proof. By $\left(3^{\prime}\right)$, with $a$ replaced by $(a, b)$ and $b$ replaced by $d$, $(a, b, d, c ; a, b)=(d, c ; a, b ; d)(c ; a, b ; d ; c)=(a, b ; d, c ; d)(a, b, c, d, c)$, so that, since $(a, b ; d, c ; d)=(a, b, d, c, d),(5)$ is true. By $\left(2^{\prime}\right)$ and $\left(3^{\prime}\right)$ with $b$ replaced by $(b, c)$ and $c$ replaced by $(d, f),(b, c, a ; d, f, a)=(a ; b, c ; d, f ; a)=$ $(b, c ; d, f ; a ; b, c)(d, f ; b, c ; a ; d, f)$, so that (6) is true. Finally, (7) and (8) are obvious from (1').

4. Lemma 4.1. If $a, b, c$ and $d$ are elements of order 2 in a group $G$ of exponent 4 , then

$$
(a, b ; c, d) \equiv(a, c ; b, d)(a, d ; b, c) \bmod G_{5} .
$$

Proof. First, working modulo $G_{5}$ and collecting as we did in the proof of Lemma 3.1 we obtain $(a b c d)^{2}=T_{2} T_{3} T_{4}$ where

$$
\begin{aligned}
& T_{2}=(a, b)(a, c)(b, c)(a, d)(b, d)(c, d) \\
& T_{3}=(a, c, b)(a, d, c)(a, d, b)(b, d, c) \\
& T_{4}=(a, d, b, c) .
\end{aligned}
$$

Note that modulo $G_{5}, T_{2}, T_{3}$ and $T_{4}$ commute, and $T_{3}^{2}=T_{4}^{2}=1$. Hence, modulo $G_{5}, 1=(a b c d)^{4}=T_{2}^{2}$. Collecting the $(a, d)$ 's in $T_{2}^{2}$ we obtain $1 \equiv X A B C Y \bmod G_{5}$, where

$$
\begin{aligned}
& X=[(a, b)(a, c)(b, c)]^{2} \\
& A=(b, c ; b, d)(b, c ; c, d)(b, d ; c, d) \\
& B=(a, c ; a, d)(a, c ; c, d)(a, d ; c, d) \\
& C=(a, b ; a, d)(a, b ; b, d)(a, d ; b, d) \\
& Y=(a, b ; c, d)(a, c ; b, d)(a, d ; b, c) .
\end{aligned}
$$

Now modulo $G_{5}, X=1$, while $A=B=C=1$ by $\left(2^{\prime}\right)$ and $\left(3^{\prime}\right)$. Hence, $1 \equiv(a, b ; c, d)(a, c ; b, d)(a, d ; b, c) \bmod G_{5}$, which is $(9)$.

CoRollary 1. If $x_{1}, \cdots, x_{k}$ and a are elements of order 2 in a group $G$ of exponent 4 , then for $i=2, \cdots, k$,

$$
\left(x_{1}, a, x_{2}, a, \cdots, x_{i}, \cdots, x_{k}\right) \equiv\left(x_{1}, x_{2}, \cdots, a, x_{i}, a, \cdots, x_{k}\right) \bmod G_{k+3} .
$$

Hence, if two of $x_{1}, \cdots, x_{k}$, a are equal, $\left(x_{1}, a, x_{2}, a, \cdots, x_{k}\right) \equiv 1 \bmod G_{k+3}$. 
Proof. Let $a, b, c$ and $d$ be elements of order 2 in $G$. Then modulo $G_{6}$,

$$
\begin{aligned}
(b, a, c, a, d) & =(b, a ; c, a ; d) \\
& =(b, a, d ; c, a)(c, a, d ; b, a) \\
& =(b, a, c ; d, a)(c, a, b ; d, a) \\
& =(b, c, a ; d, a) \\
& =(b, c, a, d, a) .
\end{aligned}
$$

The first statement follows. Now the second statement is clearly true if $a$ appears a third time, since then $\left(x_{1}, a, x_{2}, a, \cdots, a, \cdots, x_{k}\right)=$ $\left(x_{1}, x_{2}, \cdots, a, a, a, \cdots, x_{k}\right)=1$. If some $x_{i}$ appears twice, then modulo $G_{k+3}\left(x_{1}, a, x_{2}, a, \cdots, x_{i}, \cdots, x_{k}\right)=\left(x_{1}, \cdots, a, x_{i}, a, \cdots, x_{k}\right)=\left(x_{1}, x_{2}, \cdots\right.$, $\left.x_{i}, a, x_{i}, \cdots, x_{k}\right)=\left(x_{1}, x_{i} x_{2}, x_{i}, \cdots, a, \cdots, x_{k}\right)$ (the second step following from (5)), and we are back to the case of three appearances of $a$. Thus the corollary is proved.

COROLlaRY 2. If $a, b, c, d$ and $f$ are elements of order 2 in a group $G$ of exponent 4, then

$$
\begin{aligned}
& 1 \equiv(a, f, b ; c, d)(a, f, c ; b, d)(a, f, d ; b, c) \bmod G_{6} \\
& (a, c ; d, f ; b)(a, d ; c, f ; b) \equiv(c, d ; a, f ; b) \bmod G_{6} .
\end{aligned}
$$

Proof. These follow from (9).

THEOREM 4.1. $G(4)_{6}=1$.

Proof. Let the generators of $G(4)$ be $a, b, c$ and $d$ and consider any commutator $C=\left(x_{1}, x_{2}, x_{3}, x_{4}, x_{5}, x_{6}\right)$ in $a, b, c$ and $d$. It will be sufficient to prove $C=1$ under the assumption that $G(4)_{7}=1$. As in the proof of Theorem 3.1, we may suppose that $C=\left(a, x_{2}, x_{3}, x_{4}, x_{5}, a\right)$. Moreover, if $x_{2}, x_{3}, x_{4}$ or $x_{5}$ is $a$, then by Theorem 2.1 or Corollary 1 of Lemma 4.1, $C=1$. It will thus be sufficient to prove $(a, b, c, b, d, a)=1,(a, b, c, d, b ; a)=$ 1 , and $(a, c, b, d, b, a)=1$. Now by Corollary 1 of Lemma 4.1, $(a, b, c, b, d, a)=(a, c, b, d, b, a)=1$, while by $\left(1^{\prime}\right), \quad(a, b, c ; b, d, a)=$ $(a, c, b ; b, d ; a)(b, c, a ; b, d ; a)$, so that by $(6)(a, b, c ; b, d ; a)=1$. Thus $(a, b, c, d, b, a)=(a, b, c, b, d, a)(a, b, c ; b, d ; a)=1$, and the theorem is proved.

5. The main result, that $G(n)_{n+2}=1$, has now been proved for $n=2,3$ and 4 . In this section we derive an identity analogous to (1) and (9) for five generators. This identity enables us to prove, in $\S 6$, that $G(n)_{n+2}=1$ for $n \geq 5$.

Lemma 5.1. If $a, b, c, d$ and $f$ are elements of order 2 in a group $G$ of exponent 4, then 


$$
(a, b ; c, d ; f) \equiv(c, b ; f, d ; a)(f, b ; a, d ; c) \bmod G_{6} .
$$

CoROLlary. If $\left(x_{1}, \cdots, x_{k}\right),\left(y_{1}, \cdots, y_{j}\right),\left(z_{1}, \cdots, z_{m}\right), a$ and $b(k, j, m \geq$ 1) are elements of order 2 in a group $G$ of exponent 4, then

$$
\left(x_{1}, \cdots, x_{k}, a ; y_{1}, \cdots, y_{j}, b ; z_{1}, \cdots, z_{m}\right) \equiv C_{1} C_{2} \bmod G_{k+j+m+3},
$$

where

$$
\begin{aligned}
& C_{1}=\left(y_{1}, \cdots, y_{j} ; z_{1}, \cdots, z_{m} ; x_{1}, \cdots, x_{k}, b ; a\right) \\
& C_{2}=\left(x_{1}, \cdots, x_{k} ; z_{1}, \cdots, z_{m} ; y_{1}, \cdots, y_{j}, a ; b\right) .
\end{aligned}
$$

Proof of Lemma 5.1. First, working modulo $G_{5}$, we collect $f$ 's in the expression $(a b c d f)^{2}$ to get $(a b c d f)^{2}=(a b c d) a(a, f) b(b, f) c(c, f) d(d, f)$. Then collecting $b, c$ and $d$ in that order we obtain $(a b c d f)^{2}=(a b c d)^{2} S_{2} S_{3} S_{4}$ where

$$
\begin{aligned}
& S_{2}=(a, f)(b, f)(c, f)(d, f) \\
& S_{3}=(a, f, d)(a, f, c)(a, f, b)(b, f, d)(b, f, c)(c, f, d) \\
& S_{4}=(a, f, c, d)(a, f, b, d)(a, f, b, c)(b, f, c, d) .
\end{aligned}
$$

But as in the proof of Lemma $4.1,(a b c d)^{2} \equiv T_{2} T_{3} T_{4} \bmod G_{5}$, where

$$
\begin{aligned}
& T_{2}=(a, b)(a, c)(a, d)(b, d)(c, d) \\
& T_{3}=(a, c, b)(a, d, c)(a, d, b)(b, d, c) \\
& T_{4}=(a, d, b, c) .
\end{aligned}
$$

Thus, modulo $G_{5},(a b c d f)^{2}=T_{2} T_{3} T_{4} S_{2} S_{3} S_{4}$. But then, modulo $G_{6}$,

$$
\begin{aligned}
1=(a b c d f)^{4} & =T_{2} T_{3} T_{4} S_{2} S_{3} T_{2} T_{3} T_{4} S_{2} S_{3} \\
& =T_{2} T_{3} T_{4} T_{2} S_{2}\left(S_{2}, T_{2}\right) S_{3}\left(S_{3}, T_{2}\right) T_{3} T_{4} S_{2} S_{3} \\
& =\left(T_{2} T_{3} T_{4}\right)^{2} S_{2}\left(S_{2}, T_{3}\right)\left(S_{2}, T_{2}\right) S_{3}\left(S_{3}, T_{2}\right) S_{2} S_{3} \\
& =S_{2}\left(S_{2}, T_{3}\right)\left(S_{2}, T_{2}\right) S_{3}\left(S_{3}, T_{2}\right) S_{2} S_{3} \\
& =S_{2}^{2}\left(S_{2}, T_{3}\right)\left(S_{2}, T_{2}\right) S_{3}\left(S_{3}, S_{2}\right)\left(S_{3}, T_{2}\right) S_{3} \\
& =S_{2}^{2}\left(S_{2}, T_{3}\right)\left(S_{2}, T_{2}\right) S_{3}^{2}\left(S_{3}, S_{2}\right)\left(S_{3}, T_{2}\right) .
\end{aligned}
$$

But modulo $G_{6}, S_{3}^{2}=1$, while $S_{2}^{2}$ is a product of commutators of weight 4. Thus the last relation may be rewritten as $1 \equiv A \bmod G_{6}$ where $A$ is a product of commutators in $a, b, c, d$ and $f$ of weight 4 or 5 ; hence the factors of $A$ commute modulo $G_{6}$. Let $A_{a}^{\prime}$ be the product of all factors of $A$ which do not contain $a$ as argument, and let $A_{a}$ be the product of the remaining factors of $A$. Then $1 \equiv A_{a}^{\prime} A_{a} \bmod G_{b}$, so that, setting $a=1,1 \equiv A_{a}^{\prime} \bmod G_{6}$, and hence $1 \equiv A_{a} \bmod G_{6}$. Continuing this argument we finally arrive at $1 \equiv A_{\text {abcaf }} \bmod G_{6}$, where $A_{a b c a f}$ is the product of all factors of $A$ which contain each of $a, b, c, d$ and $f$. But what are 
these factors? Clearly $S_{2}^{2}$ and $\left(S_{2}, T_{2}\right)$ do not contain any such factors; and since each factor of $S_{2}$ and $S_{3}$ contains $f,\left(S_{3}, S_{2}\right)$ cannot contain any such factors. We are left with $\left(S_{2}, T_{3}\right)$ and $\left(S_{3}, T_{2}\right)$. The product of the desired factors of $\left(S_{2}, T_{3}\right)$ is clearly

$$
(a, f ; b, d, c)(b, f ; a, d, c)(c, f ; a, d, b)(d, f ; a, c, b),
$$

while the product of the desired factors of $\left(S_{3}, T_{2}\right)$ is $(a, f, d ; b, c)(a, f, c ; b, d)(a, f, b ; c, d)(b, f, d ; a, c)(b, f, c ; a, d)(c, f, d ; a, b)$.

Hence, modulo $G_{6}$,

$$
\begin{aligned}
1=(a, f ; b, d, c) & (b, f ; a, d, c)(c, f ; a, d, b)(d, f ; a, c, b) \\
& \cdot(a, f, d ; b, c)(a, f, c ; b, d)(a, f, b ; c, d) \\
& \cdot(b, f, d ; a, c)(b, f, c ; a, d)(c, f, d ; a, b) .
\end{aligned}
$$

so that by (10)

$$
\begin{array}{r}
1=(a, f ; b, d, c)(b, f ; a, d, c)(c, f ; a, d, b)(d, f ; a, c, b) \\
\cdot(b, f, d ; a, c)(b, f, c ; a, d)(c, f, d ; a, b) .
\end{array}
$$

Using (7) on the first four factors gives, modulo $G_{6}$,

$$
\begin{aligned}
1=(a, f, c ; b, d)(a, f ; b, d ; c)(b, f, c ; a, d)(b, f ; a, d ; c) \\
\cdot(c, f, b ; a, d)(c, f ; a, d ; b)(d, f, b ; a, c)(d, f ; a, c ; b) \\
\cdot(b, f, d ; a, c)(b, f, c ; a, d)(c, f, d ; a, b) \\
=(a, f, c ; b, d)(a, f ; b, d ; c)(b, f ; a, d ; c)(c, f, b ; a, d)(c, f ; a, d ; b) \\
\cdot(d, f, b ; a, c)(d, f ; a, c ; b)(b, f, d ; a, c)(c, f, d ; a, b) \\
=(a, f, c ; b, d)(a, f ; b, d ; c)(b, f ; a, d ; c)(c, f, b ; a, d) \\
\cdot(c, f ; a, d ; b)(d, f ; a, c ; b)(b, d, f ; a, c)(c, f, d ; a, b),
\end{aligned}
$$

where the last step follows from (8). Now applying (11) twice gives

$$
\begin{gathered}
1=(a, f, c ; b, d)(a, b ; d, f ; c)(c, f, b ; a, d)(a, f ; c, d ; b) \\
\cdot(b, d, f ; a, c)(c, f, d ; a, b),
\end{gathered}
$$

so that by $(10)$

$$
1=(a, f, c ; b, d)(a, b ; d, f ; c)(a, f ; c, d ; b)(b, d, f ; a, c)(c, f, a ; b, d)
$$

and hence by (8)

$$
1=(a, b ; d, f ; c)(a, f ; c, d ; b)(b, d, f ; a, c)(a, c, f ; b, d) .
$$

Thus, by (7)

$$
1 \equiv(a, b ; d, f ; c)(a, f ; c, d ; b)(a, c ; b, d ; f) \bmod G_{6},
$$


so that interchanging $a$ with $b$ and $c$ with $f$ we get

$$
1 \equiv(a, b ; c, d ; f)(c, b ; f, d ; a)(f, b ; a, d ; c) \bmod G_{6}
$$

which is (12). Thus the lemma is proved.

The corollary follows immediately.

6. Having proved the crucial relation (12), we are now in a position to prove the main theorem.

TheOREM 6.1. Let $G(n),(n=1,2, \cdots)$ be the freest group of exponent 4 generated by $n$ elements of order 2 . Then $G(n)_{n+2}=1$.

Proof. The proof is by induction on $n$. We have the result for $n=1,2,3$ and 4. Assuming the result true for $n$ we now prove it for $n+1$. As before, we may assume $G(n+1)_{n+4}=1$. Consider a commutator $C=\left(y_{1}, y_{2}, \cdots, y_{n+3}\right)$ in the generators $x_{1}, \cdots, x_{n}, a$ and $b$ of $G(n+1)$. As before, we may restrict attention to the case $C=\left(a, y_{2}, \cdots\right.$, $\left.y_{n+2}, a\right)$. There are two possibilities to consider-Case 1: $a$ appears again; Case 2: $b$ appears twice. In either case we may assume that every $x_{i}$ appears once, since otherwise, by the inductive assumption, $C=1$.

Case 1. The proof in this case is by induction on the position of the middle $a$. Clearly $\left(a, y_{2}, a, \cdots, a\right)=1$. Assume that for some $i \geq 3$, $\left(a, y_{2}, \cdots, y_{i-1}, a, \cdots, a\right)=1$. Then

$$
\begin{aligned}
\left(a, y_{2}, \cdots,\right. & \left.y_{i}, a, y_{i+1}, \cdots, y_{n+2}, a\right) \\
= & \left(a, y_{2}, \cdots, y_{i-1} ; y_{i}, a ; y_{i+1} ; \cdots ; y_{n+2} ; a\right) \\
= & \left(a, y_{2}, \cdots, y_{i-1} ; y_{i}, a ; a, y_{n+2}, \cdots, y_{i+1}\right),
\end{aligned}
$$

where the last step follows from $G(n)_{n+2}=1$. But by (13),

$$
\left(a, y_{2}, \cdots, y_{i-1} ; y_{i}, a ; a, y_{n+2}, \cdots, y_{i+1}\right)=C_{1} C_{2}
$$

where

$$
\begin{aligned}
& C_{1}=\left(a, y_{2}, \cdots, y_{i-2}, y_{i} ; a, y_{n+2}, \cdots, y_{i+1}, a ; y_{i-1}\right) \\
& C_{2}=\left(a, y_{2}, \cdots, y_{i-2} ; a, y_{n+2}, \cdots, y_{i+1} ; y_{i-1}, a ; y_{i}\right)
\end{aligned}
$$

Since $y_{i}$ and $y_{i-1}$ appear only once, by the assumption that $G(n)_{n+2}=1$ we have $C_{1}=C_{2}=1$. Hence, by induction, $C=1$ if $a$ appears three times.

Case 2. In this case also the proof is by induction, this time on the distance between the $b$ 's. Let

$$
C=\left(a, z_{1}, \cdots, z_{i}, b, z_{i+1}, \cdots, z_{j}, b, z_{j+1}, \cdots, z_{n-1}, a\right),
$$

where $0 \leq i<j \leq n-1$ (that is, there might be no entries between 
the $a$ 's and the $b$ 's). If $j-i=1$, then clearly $C=1$. Assume that $C=1$ for $j-i=k \geq 1$. Then as in Case 1 ,

$$
\begin{aligned}
\left(a, z_{1}, \cdots, z_{i}, b, z_{i+1}, \cdots, z_{i+k+1}, b, z_{i+k+2}, \cdots, z_{n-1}, a\right) \\
\quad=\left(a, z_{1}, \cdots, z_{i}, b, z_{i+1}, \cdots, z_{i+k} ; z_{i+k+1}, b ; a, z_{n-1}, \cdots, z_{i+k+2}\right) \\
\quad=C_{1} C_{2}
\end{aligned}
$$

where

$$
\begin{aligned}
& C_{1}=\left(a, \cdots, b, \cdots, z_{i+k-1}, z_{i+k+1} ; a, z_{n-1}, \cdots, z_{i+k+2}, b ; z_{i+k}\right)=1 \\
& C_{2}=\left(a, \cdots, b, \cdots, z_{i+k-1} ; a, z_{n-1}, \cdots, z_{i+k+2} ; z_{i+k}, b ; z_{i+k+1}\right)=1
\end{aligned}
$$

Thus $C=1$ for $j-i=k+1$, so that by induction $C=1$ if $b$ appears twice.

Since $C=1$ in both cases, we conclude that $G(n+1)_{n+3}=1$, so that by induction $G(n)_{n+2}=1$ for $n=1,2, \cdots$.

7. The author conjectures that the class of $G(n)$ is precisely $n+1$ for $n>2$. As supporting evidence, he has constructed $G(n) / G(n)^{\prime \prime}$ and shown that its class is exactly $n$. Moreover, for $n=3$ and $n=4$, $G(n)^{\prime \prime}$ is fairly large, and $G(n)_{n+1} \neq 1$.

\section{BIBLIOGRAPHY}

1. M. Hall, Jr., The Theory of Groups, the Macmillan Co., 1959.

2. I. N. Sanov, Solution of Burnside's problem for exponent 4, Leningrad State Univ. Ann. 10, (1940), 166-170. 



\section{PACIFIC JOURNAL OF MATHEMATICS}

\section{EDITORS}

\author{
David Gilbarg \\ Stanford University \\ Stanford, California \\ F. H. Brownell \\ University of Washington \\ Seattle 5 , Washington
}

\author{
A. L. Whiteman \\ University of Southern California \\ Los Angeles 7, California \\ L. J. PAIGE \\ University of California \\ Los Angeles 24, California
}

\section{ASSOCIATE EDITORS}

\author{
E. F. BECKENBACH \\ T. M. CHERRY \\ D. DERRY
}
E. HEWITT
A. HORN
L. NACHBIN
M. OHTSUKA
H. L. ROYDEN
M. M. SCHIFFER

E. SPANIER

E. G. STRAUS

F. WOLF

\section{SUPPORTING INSTITUTIONS}

\author{
UNIVERSITY OF BRITISH COLUMBIA \\ CALIFORNIA INSTITUTE OF TECHNOLOGY \\ UNIVERSITY OF CALIFORNIA \\ MONTANA STATE UNIVERSITY \\ UNIVERSITY OF NEVADA \\ NEW MEXICO STATE UNIVERSITY \\ OREGON STATE COLLEGE \\ UNIVERSITY OF OREGON \\ OSAKA UNIVERSITY \\ UNIVERSITY OF SOUTHERN CALIFORNIA
}

\author{
STANFORD UNIVERSITY \\ UNIVERSITY OF TOKYO \\ UNIVERSITY OF UTAH \\ WASHINGTON STATE COLLEGE \\ UNIVERSITY OF WASHINGTON

AMERICAN MATHEMATICAL SOCIETY
CALIFORNIA RESEARCH CORPORATION
HUGHES AIRCRAFT COMPANY
SPACE TECHNOLOGY LABORATORIES
NAVAL ORDNANCE TEST STATION

Mathematical papers intended for publication in the Pacific Journal of Mathematics should be typewritten (double spaced), and the author should keep a complete copy. Manuscripts may be sent to any one of the four editors. All other communications to the editors should be addressed to the managing editor, L. J. Paige at the University of California, Los Angeles 24, California. ..

50 reprints per author of each article are furnished free of charge; additional copies may be obtained at cost in multiples of 50 .

The Pacific Journal of Mathematics is published quarterly, in March, June, September, and December. The price per volume (4 numbers) is $\$ 12.00$; single issues, $\$ 3.50$. Back numbers are available. Special price to individual faculty members of supporting institutions and to individual members of the American Mathematical Society: $\$ 4.00$ per volume; single issues, $\$ 1.25$.

Subscriptions, orders for back numbers, and changes of address should be sent to Pacific Journal of Mathematics, 2120 Oxford Street, Berkeley 4, California.

Printed at Kokusai Bunken Insatsusha (International Academic Printing Co., Ltd.), No. $6_{4}$ 2-chome, Fujimi-cho, Chiyoda-ku, Tokyo, Japan.

PUBLISHED BY PACIFIC JOURNAL OF MATHEMATICS, A NON-PROFIT CORPORATION

The Supporting Institutions listed above contribute to the cost of publication of this Journăl, but they are not owners or publishers and have no responsibility for its content or policies. 


\section{Pacific Journal of Mathematics}

\section{Vol. 10, No. $3 \quad$ November, 1960}

Glen Earl Baxter, An analytic problem whose solution follows from a simple

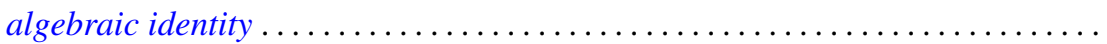

Leonard D. Berkovitz and Melvin Dresher, A multimove infinite game with linear payoff. .

Earl Robert Berkson, Sequel to a paper of A. E. Taylor ......................

Gerald Berman and Robert Jerome Silverman, Embedding of algebraic systems.... 767

Peter Crawley, Lattices whose congruences form a boolean algebra . . . . . ...... 777

Robert E. Edwards, Integral bases in inductive limit spaces . . . . . . . . . . . . . . .

Daniel T. Finkbeiner, II, Irreducible congruence relations on lattices . . . . . . . . . .

William James Firey, Isoperimetric ratios of Reuleaux polygons . . . . . . . . . . . 787

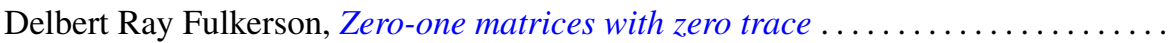

Leon W. Green, A sphere characterization related to Blaschke's conjecture........

Israel (Yitzchak) Nathan Herstein and Erwin Kleinfeld, Lie mappings in

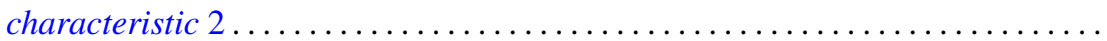

Charles Ray Hobby, A characteristic subgroup of a $p$-group .................

R. K. Juberg, On the Dirichlet problem for certain higher order parabolic

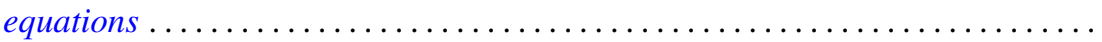

Melvin Katz, Infinitely repeatable games ......................

Emma Lehmer, On Jacobi functions . . . . . . . . . . . . . . . . . . . . . . . . .

D. H. Lehmer, Power character matrices

Henry B. Mann, A refinement of the fundamental theorem on the density of the sum of two sets of integers.

Marvin David Marcus and Roy Westwick, Linear maps on skew symmetric matrices: the invariance of elementary symmetric functions . .

Richard Dean Mayer and Richard Scott Pierce, Boolean algebras with ordered

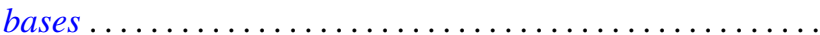

Trevor James McMinn, On the line segments of a convex surface in $E_{3} \ldots$

Frank Albert Raymond, The end point compactification of manifolds ..

Edgar Reich and S. E. Warschawski, On canonical conformal maps of regions of arbitrary connectivity

Marvin Rosenblum, The absolute continuity of Toeplitz's matrices...

Lee Albert Rubel, Maximal means and Tauberian theorems . .

Helmut Heinrich Schaefer, Some spectral properties of positive linear operators

Jeremiah Milton Stark, Minimum problems in the theory of pseudo-conformal transformations and their application to estimation of the curvature of the invariant metric.

Robert Steinberg, The simplicity of certain groups ...

Hisahiro Tamano, On paracompactness. .

Angus E. Taylor, Mittag-Leffler expansions and spectral theory .

Marion Franklin Tinsley, Permanents of cyclic matrices ...... . 